\title{
Posttraumatic Embitterment Disorder in Patients with Chronic Kidney Disease
}

\author{
Kyungsoo Lee ${ }^{1}$, Ho Chul Song ${ }^{1}$, Euy Jin Choi ${ }^{1}$, Chi-Un Pae ${ }^{2,3,4}$, Yong Kyun Kim ${ }^{1,4}$ \\ Departments of ${ }^{1}$ Internal Medicine and ${ }^{2}$ Psychiatry, College of Medicine, The Catholic University of Korea, Seoul, Korea, ${ }^{3}$ Department of \\ Psychiatry and Behavioral Sciences, Duke Medical Center, Durham, NC, USA, ${ }^{4}$ Cell Death Disease Research Center, College of Medicine, \\ The Catholic University of Korea, Seoul, Korea
}

\begin{abstract}
Objective: Posttraumatic embitterment disorder (PTED), a subgroup of an adjustment disorder, is a feeling with anger and helplessness. Hemodialysis may be a trigger event leading to PTED. We investigated the prevalence of PTED in patients with each categorized stages of chronic kidney disease (CKD) and the association between PTED and depression and functional impairment.

Methods: Patients were categorized into three groups according to the stages of CKD (stage I-II, III-IV, and V). CKD (I-II) group was defined as estimated glomerular filtration rate (eGFR) $>60 \mathrm{ml} / \mathrm{min} / 1.73 \mathrm{~m}^{2}$, CKD (III-IV) group as eGFR $<60 \mathrm{ml} / \mathrm{min} / 1.73 \mathrm{~m}^{2}$, and CKD (V) group as CKD stage $\mathrm{V}$ including patients ongoing hemodialysis. Patients were assessed for the prevalence of PTED, depression, and decreased quality of life by using the scale of PTED, Patient Health Questionnaire-9 (PHQ-9), and EuroQol Five Dimensional Questionnaires, Visual Analogue Scale (EQ-5D-VAS), respectively.

Results: A total of 445 patients were analyzed. The number of patients in CKD (I-II) was 166, CKD (III-IV) was 172, and CKD (V) was 107. Multivariate analysis by binomial logistic regression demonstrated that CKD (V) was significantly associated with the prevalence of PTED (odds ratio, 4.13; 95\% confidence interval, 1.56-15.6; $p=0.006$ ) after adjustment for age, gender, and diabetes mellitus. Also, a significant correlation existed between PTED and EQ-5D-VAS in all stages, but the correlation was nonsignificant between PTED and PHQ-9 score in group CKD (V).

Conclusion: The findings suggest that PTED is underdiagnosed in CKD patients. Acknowledgment and diagnosis of PTED in CKD patients may lead to a better quality of life.
\end{abstract}

KEY WORDS: Posttraumatic embitterment disorder; Dialysis; Depression; Quality of life; Chronic renal insufficiency.

\section{INTRODUCTION}

Hemodialysis is the endpoint in chronic kidney disease (CKD). Patients often look back a long history of dealing

Received: November 8, 2017 / Revised: November 14, 2017

Accepted: November 21, 2017

Address for correspondence: Chi-Un Pae, MD, PhD

Department of Psychiatry, Bucheon St. Mary's Hospital, College

of Medicine, The Catholic University of Korea, 327 Sosa-ro,

Wonmi-gu, Bucheon 14647, Korea

Tel: +82-32-340-2140, Fax: +82-32-340-2544

E-mail: pae@catholic.ac.kr

ORCID: https://orcid.org/0000-0003-1632-4248

Yong Kyun Kim, MD, PhD

Department of Internal Medicine, Bucheon St. Mary's Hospital,

College of Medicine, The Catholic University of Korea, 327

Sosa-ro, Wonmi-gu, Bucheon 14647, Korea

Tel: +82-32-340-2140, Fax: +82-32-340-2544

E-mail: drkimyk@catholic.ac.kr

ORCID: https://orcid.org/0000-0002-1871-3549 with their illness. This includes the question why they are the ones who are hit by the illness, but also whether physicians or the health system did what could have been done, why they do not get a kidney transplant, or how the family or the employer reacts to their impairment. ${ }^{1)}$ It can be expected that there are some patients who feel that they have been let behind, that they have been treated unfair and that their fate is unjust. They have reasons to look back in anger and sometimes also may feel stimulated to fight back against the world, while they are helpless and hopeless. In clinical practice these patients give the impression of being embittered. ${ }^{2)}$

Embitterment is an emotion which is known to everybody in reaction to injustice and being let behind. ${ }^{3)}$ It is associated with a feeling of being a "loser", of being victimized, of anger, or helplessness and hopelessness, and

(ㄷ) This is an Open-Access article distributed under the terms of the Creative Commons Attribution Non-Commercial License (http://creativecommons.org/licenses/by-nc/4.0) which permits unrestricted non-commercial use, distribution, and reproduction in any medium, provided the original work is properly cited. 
especially of phantasies of aggression or revenge, be it against other persons or the fate and God himself. In greater intensity, it can become pathological, similar to anxiety. This state of mind has been described unter the heading of "posttraumatic embitterment disorder". ${ }^{4-8)}$

Previous studies suggest that hemodialysis patients suffer to an increased rate from depression, anxiety, or psychological impairment in general and that this is associated with a higher risk of morbidity and mortality. ${ }^{9-11)}$ Embitterment has so far not been taken into account, although the clinical impression is that this may be of relevance. Therefore the purpose of this study was to assess the prevalence of embitterment in CKD patients, including those on long-term hemodialysis, and investigate its correlation with quality of life and mood impairment in general.

\section{METHODS}

\section{Study Population and Data Collection}

The total number of patient selected was 445 . Among 445 patients, 166 CKD stage (I-II) patients with estimated glomerular filtration rate (eGFR) $>60 \mathrm{ml} / \mathrm{min} / 1.73 \mathrm{~m}^{2}$, 172 CKD stage (III-IV) patients (eGFR, $<60 \mathrm{ml} / \mathrm{min} / 1.73$ $\left.\mathrm{m}^{2}\right)$, and 107 CKD stage $(\mathrm{V})$ patients with patients ongoing hemodialysis. Patients needed to be 17 years or older and without severe psychiatric disorder to be enrolled. Questionnaires and clinical information was received at the point of registration. Informed consent (IC) was received from all participants after full explanation. The study protocol and IC were fully reviewed and approved by the Institutional Review Board (IRB) of The Catholic University of Korea, Bucheon St. Mary's Hospital (IRB No. HC14OISI0054).

\section{Measurements}

Posttraumatic embitterment disorder (PTED) self-rating scale

The scale of PTED is a 19-item questionnaire which starts with the statement: "During the last years there was a severe and negative life event $\cdots$ " followed by single items like " $\cdots$ that hurt my feelings and caused consid-

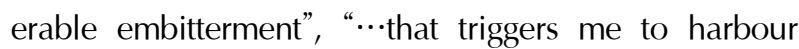
thoughts of revenge", or " $\cdots$ which frequently evokes painful memories". Answers are given on Likert scale from (0) not at all true toe (4) extremely true. The sum score can be interpreted as measure of reactive embitterment. Higher scores are indicative of PTED. We have used the Korean version of PTED scale which had been reported to stable and reliable. ${ }^{4)}$ Score greater than 30 is to suspect PTED and therefore we have used the cut-off score of $30 .{ }^{12)}$

The Patient Health Questionnaire-9 (PHQ-9)

PHQ-9 is a 9-item screening questionnaire asking for symptoms of depression in various clinical population which has also been validated and commonly used in clinical practice in Korea. ${ }^{4,13-19)}$ As each item is rated on a scale from 0 to 3 , the total score ranges from 0 to 27 .

EuroQol Five Dimensional Questionnaires, Visual Analogue Scale (EQ-5D-VAS)

EQ-5D is one of the most frequently used scale to assess patients' health-related quality of life. ${ }^{20,21)}$ We proceeded the questionnaire with a visual analogue scale from 0 "worst" to a 100 "best". Respondents were to express their current health status by marking a point between the scales.

\section{Statistical Analysis}

All data were expressed in mean \pm standard deviation (SD) unless otherwise specified. Median with ranges was presented in those without normal distribution. In continuous variables, Student's $t$ test, Mann-Whitney test, one-way ANOVA test were used as appropriate. Pearson's chi-square test or Fisher's exact test was used to analyze the differences in categorical variables. A $p$ value of $<0.05$ were considered significant. Univariate logistic regression identified variables that were related with the prevalence of PTED, and those with $p<0.1$ were included in multivariate analysis. Univariate and multiple linear regression analyses were done to examine the relationship between the scale of PTED, PHQ-9, and EQ-5D-VAS score. All analysis was done by SPSS version 11.0 (SPSS Inc., Chicago, IL, USA).

\section{RESULTS}

\section{Baseline Demographic and Clinical Characteristics}

Baseline characteristics of the study population according to CKD stage are shown in Table 1.

The total number of patient was 445; 166 in the CKD (I-II), 172 in the CKD (III-IV), and 107 patients CKD (V). 
Table 1. Baseline characteristics according to CKD stage

\begin{tabular}{|c|c|c|c|c|}
\hline \multirow{2}{*}{ Characteristic } & \multicolumn{3}{|c|}{ CKD stage } & \multirow{2}{*}{$p$ value } \\
\hline & I, II (n=166) & III, IV (n=172) & $V(n=107)$ & \\
\hline Age (yr) & $40.82 \pm 13.4$ & $57.9 \pm 11.2$ & $59.4 \pm 12.8$ & $<0.001$ \\
\hline Sex, male & $61(36.7)$ & $105(64.5)$ & $54(50.5)$ & $<0.001$ \\
\hline Body mass index $\left(\mathrm{kg} / \mathrm{m}^{2}\right)$ & $24.0 \pm 3.66$ & $24.2 \pm 3.72$ & $22.8 \pm 4.11$ & 0.009 \\
\hline Diabetes mellitus & $17(10.2)$ & $117(68.0)$ & $70(65.4)$ & $<0.001$ \\
\hline Systolic BP (mmHg) & $114 \pm 11$ & $124 \pm 18$ & $140 \pm 19$ & $<0.001$ \\
\hline Diastolic BP (mmHg) & $72 \pm 8$ & $71 \pm 12$ & $78 \pm 13$ & $<0.001$ \\
\hline Hemoglobin (g/dL) & $13.6 \pm 1.7$ & $12.2 \pm 1.9$ & $10.9 \pm 1.5$ & $<0.001$ \\
\hline Serum AST (IU/L) & $22.2 \pm 8.7$ & $24.5 \pm 15.7$ & $20.1 \pm 17.9$ & 0.039 \\
\hline Serum ALT (IU/L) & $23.4 \pm 18.7$ & $23.8 \pm 19.6$ & $18.0 \pm 19.1$ & 0.031 \\
\hline Serum calcium (mg/dl) & $9.3 \pm 0.6$ & $8.9 \pm 0.6$ & $9.1 \pm 1.3$ & $<0.001$ \\
\hline Serum phosphorus (mg/dl) & $3.7 \pm 0.6$ & $3.8 \pm 0.9$ & $4.8 \pm 1.6$ & $<0.001$ \\
\hline Serum albumin $(g / d l)$ & $4.2 \pm 0.7$ & $4.0 \pm 0.7$ & $5.1 \pm 11.2$ & 0.315 \\
\hline Serum TC (mg/dl) & $197.2 \pm 73.3$ & $180.7 \pm 66.9$ & $150.8 \pm 51.5$ & $<0.001$ \\
\hline Serum TG $(\mathrm{mg} / \mathrm{dl})$ & $165.3 \pm 202.6$ & $167.3 \pm 136.2$ & $116.9 \pm 91.3$ & 0.018 \\
\hline Serum creatinine (mg/dl) & $0.8 \pm 0.21$ & $1.97 \pm 0.87$ & $9.68 \pm 13.3$ & $<0.001$ \\
\hline $\mathrm{eGFR}\left(\mathrm{ml} / \mathrm{min} / 1.73 \mathrm{~m}^{2}\right)$ & $95.4 \pm 23.6$ & $36.7 \pm 13.5$ & $7.6 \pm 5.6$ & $<0.001$ \\
\hline Serum hsCRP (mg/L) & $1.0 \pm 2.6$ & $1.9 \pm 2.7$ & $3.2 \pm 6.6$ & $<0.001$ \\
\hline Free T4 (pg/ml) & $12.2 \pm 1.9$ & $12.0 \pm 2.8$ & $16.0 \pm 26.9$ & 0.155 \\
\hline $\mathrm{TSH}(\mathrm{mlU} / \mathrm{L})$ & $3.3 \pm 6.1$ & $4.2 \pm 11.7$ & $3.0 \pm 2.5$ & 0.656 \\
\hline PTED score & $6(1-14)$ & $6(1-15)$ & $10(1-29)$ & $<0.001$ \\
\hline PHQ-9 & $3(1-4)$ & $3(1-5)$ & $4(2-7)$ & $<0.001$ \\
\hline EQ-5D-VAS & $70(60-80)$ & $60(50-70)$ & $70(50-80)$ & 0.010 \\
\hline
\end{tabular}

Values are presented as mean \pm standard deviation, number $(\%)$, or median (range).

CKD, chronic kidney disease; BP, blood pressure; AST, aspartate aminotransferase; ALT, alanine aminotransferase; TC, total cholesterol; TG, triglyceride; eGFR, estimated glomerular filtration rate; hsCRP, high-sensitivity C-reactive protein; TSH, thyroid stimuating hormone; PTED, posttraumatic embitterment disorder; PHQ-9, Patient Health Questionnaire-9; EQ-5D-VAS, EuroQol Five Dimensional Questionnaires, Visual Analogue Scale.

Table 2. Logistic regression analysis for predicting prevalence of PTED

\begin{tabular}{|c|c|c|c|c|}
\hline \multirow{2}{*}{ Variable } & \multicolumn{2}{|c|}{ Univariate } & \multicolumn{2}{|c|}{ Multivariate } \\
\hline & OR $(95 \% \mathrm{Cl})$ & $p$ value & OR $(95 \% \mathrm{Cl})$ & $p$ value \\
\hline CKD V (vs. CKD I-IV) & $5.13(2.69-9.75)$ & $<0.001$ & $4.94(1.56-15.6)$ & 0.006 \\
\hline Age (per 10 yr) & $0.99(0.97-1.01)$ & 0.517 & & \\
\hline Male (vs. female) & $0.87(0.47-1.63)$ & 0.429 & & \\
\hline $\mathrm{BMI}\left(\right.$ per $\left.1 \mathrm{~kg} / \mathrm{m}^{2}\right)$ & $0.95(0.87-1.03)$ & 0.214 & & \\
\hline DM (vs. non-DM) & $1.63(0.87-3.06)$ & 0.127 & & \\
\hline Systolic BP (per $10 \mathrm{mmHg}$ ) & $1.02(1.01-1.04)$ & 0.004 & $1.00(0.98-1.02)$ & 0.895 \\
\hline Diastolic BP (per 10 mmHg) & $1.03(1.01-1.06)$ & 0.012 & $1.02(0.99-1.05)$ & 0.253 \\
\hline Hemoglobin (per $1 \mathrm{~g} / \mathrm{dl}$ ) & $0.85(0.72-1.00)$ & 0.046 & $1.05(0.86-1.29)$ & 0.604 \\
\hline Serum AST (per 1 IU/L) & $0.97(0.94-1.01)$ & 0.161 & & \\
\hline Serum calcium (per 1 mg/dl) & $1.46(0.94-2.26)$ & 0.089 & & \\
\hline Serum phosphorus (per $1 \mathrm{mg} / \mathrm{dl}$ ) & $1.35(1.08-1.68)$ & 0.009 & $1.05(0.82-1.36)$ & 0.690 \\
\hline Serum albumin (per $1 \mathrm{~g} / \mathrm{dl}$ ) & $0.98(0.88-1.11)$ & 0.808 & & \\
\hline Serum TC (per 1 mg/dl) & $0.99(0.98-1.00)$ & 0.003 & $0.99(0.99-1.00)$ & 0.101 \\
\hline Serum TG (per 1 mg/dl) & $1.00(0.99-1.00)$ & 0.036 & $1.00(0.99-1.00)$ & 0.456 \\
\hline eGFR (per $1 \mathrm{ml} / \mathrm{min} / 1.73 \mathrm{~m}^{2}$ ) & $0.99(0.98-1.00)$ & 0.004 & $1.00(0.99-1.02)$ & 0.396 \\
\hline Serum hsCRP (per 1 mg/L) & $1.03(0.97-1.01)$ & 0.347 & & \\
\hline Free T4 (per 1 pg/ml) & $1.19(0.97-1.45)$ & 0.095 & & \\
\hline TSH (per 1 mIU/L) & $0.99(0.92-1.07)$ & 0.833 & & \\
\hline
\end{tabular}

PTED, posttraumatic embitterment disorder; OR, odds ratio; 95\% Cl, 95\% confidence interval; CKD, chronic kidney disease; BMI, body mass index; DM, diabetes mellitus; BP, blood pressure; AST, aspartate aminotransferase; TC, total cholesterol; TG, triglyceride; eGFR, estimated glomerular filtration rate; hsCRP, high-sensitivity C-reactive protein; TSH, thyroid stimuating hormone. 
The mean age of patients was $51.9 \pm 15.1$ years and the mean eGFR was $51.6 \pm 39.4 \mathrm{ml} / \mathrm{min} / 1.73 \mathrm{~m}^{2}$. Among CKD (V), 54 (50.5\%) were male with a mean age of $59.4 \pm 12.8$ (SD) years. The mean duration of hemodialysis was 57 months. Seventy (65.4\%) had diabetes mellitus and 101 $(94.4 \%)$ had hypertension in CKD (V). Multivariate analysis by binomial logistic regression demonstrated that CKD (V) was significantly associated with the prevalence of PTED (odds ratio, 4.13; 95\% confidence interval, $1.56-15.6 ; p=0.006$ ) after adjustment for age, gender, and diabetes mellitus (Table 2).

\section{PTED Prevalence}

Cut-off value of 30 was used to suspect PTED. PTED score had a median score of 6 (1-14) in CKD (I-II) group, 6 (1-15) in CKD (III-IV) group, and 10 (1-29) in the CKD (V) group. Twelve (7.2\%), 7 (4.1\%), 25 (23.4\%) patients had a score above 30 in group CKD (I-II), CKD (III-IV), CKD (V), respectively (Fig. 1).

\section{A}

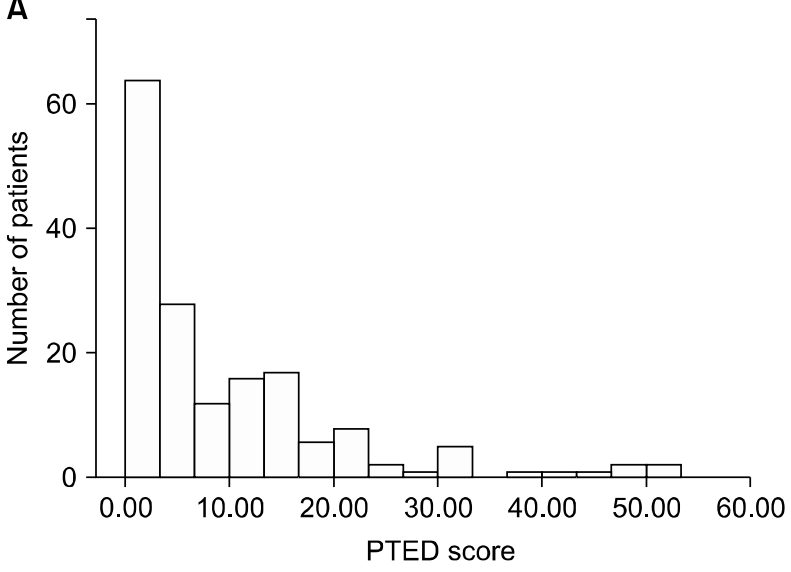

C

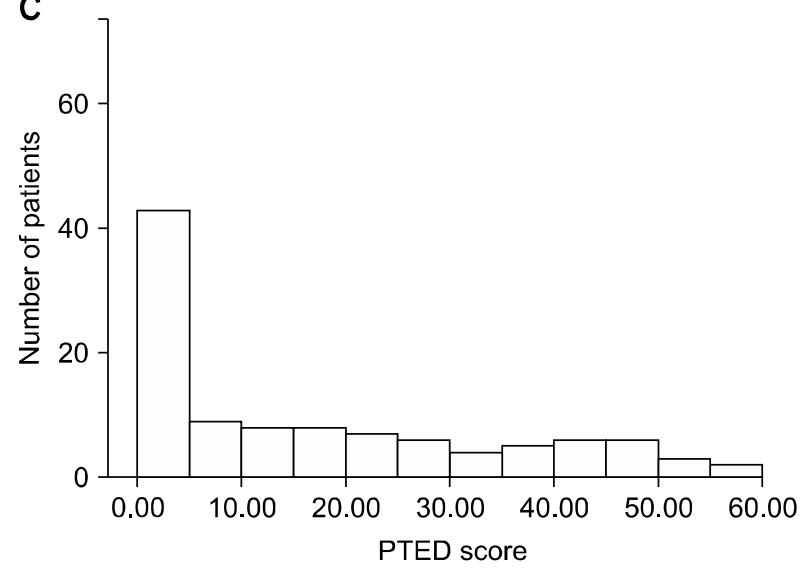

\section{Depression Score (PHQ-9) and PTED}

PHQ-9 median score was 3 (1-4), 3 (1-5), 4 (2-7) in CKD (I-II), CKD (III-IV), and CKD (V) respectively. For CKD (V) group, the linear regression coefficient of PTED score in relation to $\mathrm{PHQ}-9$ score was 0.146 in univariate linear regression and $0.188(p=0.130)$ in multivariate linear regression after adjusting for age, sex, and diabetes. CKD (I-II) and CKD (III-IV) group showed a significant positive correlation $(p<0.001$, Table 3).

\section{Relationship bewteen PTED and Quality of Life (EQ-5D-VAS)}

EQ-5D-VAS score was used to assess the quality of life. PTED score had a median score of 70 (60-80) in CKD (I-II) group, 60 (50-70) in CKD (III-IV) group, and 70 (50-80) in the CKD (V) group. For all stages of CKD, PTED score had a negative linear significant correlation to EQ-5D-VAS score $(p<0.001$, Table 3$)$.

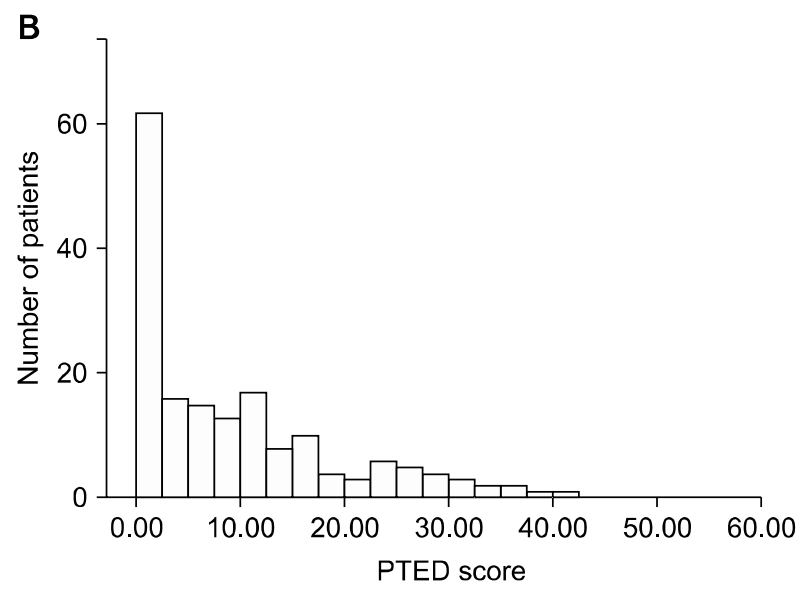

Fig. 1. Distribution of posttraumatic embitterment disorder (PTED) score according to chronic kidney disease (CKD) stage. (A) Stage I-II, (B) stage III-IV, (C) stage V. 
Table 3. Univariate and multivariate linear regression analyses for PTED score according to CKD stage

\begin{tabular}{|c|c|c|c|c|c|c|c|c|c|c|c|c|}
\hline & \multicolumn{6}{|c|}{ Univariate } & \multicolumn{6}{|c|}{ Multivariate* } \\
\hline & \multicolumn{2}{|c|}{ CKD I, II } & \multicolumn{2}{|c|}{ CKD III, IV } & \multicolumn{2}{|c|}{ CKD V } & \multicolumn{2}{|c|}{ CKD I, II } & \multicolumn{2}{|c|}{ CKD III, IV } & \multicolumn{2}{|c|}{ CKD V } \\
\hline & $\beta$-coefficient & $p$ & $\beta$-coefficient & $p$ & $\beta$-coefficient & $p$ & $\beta$-coefficient & $p$ & $\beta$-coefficient & $p$ & $\beta$-coefficient & $p$ \\
\hline PHQ-9 & 0.646 & $<0.001$ & 0.617 & $<0.001$ & 0.146 & 0.243 & 0.658 & $<0.001$ & 0.623 & $<0.001$ & 0.188 & 0.130 \\
\hline EQ-5D-VAS & -0.240 & 0.002 & -0.280 & $<0.001$ & -0.390 & $<0.001$ & -0.238 & 0.002 & -0.262 & 0.001 & -0.387 & $<0.001$ \\
\hline
\end{tabular}

PTED, posttraumatic embitterment disorder; CKD, chronic kidney disease; PHQ-9, Patient Health Questionnaire-9; EQ-5D-VAS, EuroQol Five Dimensional Questionnaires, Visual Analogue Scale.

*Adjusted for age, sex, diabetes mellitus.

\section{DISCUSSION}

As far as we can tell, this is the first journal reporting the prevalence of PTED in CKD or hemodialysis patients. PTED is a relatively new concept compared to the wellknown posttraumatic stress disorder (PTSD). There had been a report on hemodialysis patients with PTSD. ${ }^{11}$ However, we give strength to our report to that "hemodialysis" is a stressful event but not a life-threatening event as which PTSD defines. Previous report did suggest natural disaster, accidents, or fire as the stressful event but the majority was life threatening illness (66.7\%). Therefore, we would like to introduce PTED, which may lead us to a better general care in hemodialysis patients.

Embitterment is similar to depression. ${ }^{4,6,15)}$ However, in our data, the score of PTED and PHQ-9 (depression score) did not show a correlation $(p=0.242)$ in hemodialysis patients. We suggest this is due to the contrary point between depression and PTED. In contrast to depression, PTED is triggered by an unpleasant memory or event. ${ }^{6}$ Continuous exposure to the memory would aggravate the emotional instability. The problem starts in CKD patients when it comes to a start in hemodialysis. Patients are exposed to this event every other day once they have started. While most patients are tolerable to the event since they had been announced previously in the clinics for months, for those who are without insight or unprepared, initiating dialysis can be a unpleasant negative life event. Such negative life event will be continuously and inevitably exposed to the patient. The patient would feel helpless towards this life cycle. Therefore, acknowledgement towards those who satisfy the PTED score and receive hemodialysis should need a better supportive care. According to our data, presence of CKD did not increase the prevalence of depression or PTED. In contrast, CKD patients whom started hemodialysis increased significantly compared to the control group $(p<0.01)$. This data supports the fact that hemodialysis does give a significant negative emotional impact in CKD patients.

Anger is an emotion towards someone or some other event. In PTED, the anger is usually toward oneself. Self-blaming and the feeling of injustice is another important feature of PTED. ${ }^{5,6,12)}$ Hemodialysis patients, who scored more than 30 in the PTED scale, commonly regretted and was angered at their previous healthcare. Most regrets were on their poor medical compliance or unnutrional diet whether it may be true or not. Also, during the questionnaire, we realized that patients with a high score of PTED felt injustice related to their unemployment after initiating hemodialysis. Quality of life was evaluated with the correlation of PTED score. EQ-5DVAS score showed a negative correlation with the PTED score $(\beta=-0.455, p<0.001)$. Unemployment, self-blaming, injustice, and feeling of helpless would all have impacted the lowering of EQ-5D-VAS score. ${ }^{21)}$

Despite embitterment is characterized by feelings of hopelessness, fatalistic attitudes of the patients, rejection of help and an aggressive attitude in patient, needing intensive engagement of specialists those have expertise, experiences, and knowledge in this field and thus appropriate and proper handling of embitterment is huge task and barrier to clinicians in routine practice. Wisdom therapy, cognitive behavioral therapy, well-being psychology approach, establishment of prudent coping strategy, active engagement of patients, strengthening of social competency, hedonia therapy, and finally some relaxing biological agents would be helpful to manage embitterment of medical patients, although there have been no firmly approved, specific and established treatments till today. ${ }^{22)}$

One of the limitations of this study is the lack of other 
reported journals related to PTED. PTED is a relatively new concept that yet has not been introduced to other clinical areas other than psychology. More clinical reports in the future will emphasize the importance of PTED. The other limitation is a single-center limited study. This localized design allowed us to process the study in speed, but lacks support of generalizability.

PTED is an adjustment disorder that can be triggered by a negative life event like hemodialysis. The prevalence had been underdiagnosed in hemodialysis patients (23.4\%). This psychopathological condition decreases the quality of life in ESRD patients. Therefore, more acknowledgements towards this disorder may prevent its occurrence by giving better emotional support and positive images when one reaches a start in hemodialysis.

\section{Acknowledgments}

This research was supported by Basic Science Research Program through the National Research Foundation of Korea (NRF) funded by the Ministry of Education, Science and Technology (NRF-2015R1D1A1A09059195). The authors have no financial conflict of interest to declare.

\section{REFERENCES}

1. Li YN, Shapiro B, Kim JC, Zhang M, Porszasz J, Bross R, et al. Association between quality of life and anxiety, depression, physical activity and physical performance in maintenance hemodialysis patients. Chronic Dis Trans/ Med 2016;2: 110-119.

2. Joe S, Lee JS, Kim SY, Won SH, Lim JS, Ha KS. Posttraumatic embitterment disorder and hwa-byung in the general Korean population. Psychiatry Investig 2017;14:392-399.

3. Linden M, Maercker A. Embitterment. Societal, psychological, and clinical perspectives. Wien:Springer;2011.

4. Shin $\mathrm{C}$, Han C, Linden M, Chae JH, Ko YH, Kim YK, et al. Standardization of the Korean version of the posttraumatic embitterment disorder self-rating scale. Psychiatry Investig 2012;9:368-372.

5. Blom D, Thomaes S, Bijlsma JW, Geenen R. Embitterment in patients with a rheumatic disease after a disability pension examination: occurrence and potential determinants. Clin Exp Rheumatol 2014;32:308-314.

6. Linden M. Posttraumatic embitterment disorder. Psychother Psychosom 2003;72:195-202.

7. Lee JO, Kim JW, Kang HJ, Hong JP, Kim JM. Predictors of cognitive improvement during 12 weeks of antidepressant treatment in patients with major depressive disorder. Clin Psychopharmacol Neurosci 2018;16:461-468.

8. Jeon SW, Han C, Ko YH, Yoon SY, Pae CU, Choi J, et al.
Measurement-based treatment of residual symptoms using clinically useful depression outcome scale: Korean validation study. Clin Psychopharmacol Neurosci 2017;15:28-34.

9. Lopes AA, Albert JM, Young EW, Satayathum S, Pisoni RL, Andreucci VE, et al. Screening for depression in hemodialysis patients: associations with diagnosis, treatment, and outcomes in the DOPPS. Kidney Int 2004;66:2047-2053.

10. Kalantar-Zadeh K, Kopple JD, Block G, Humphreys MH. Association among SF36 quality of life measures and nutrition, hospitalization, and mortality in hemodialysis. I Am Soc Nephrol 2001;12:2797-2806.

11. Tagay S, Kribben A, Hohenstein A, Mewes R, Senf W. Posttraumatic stress disorder in hemodialysis patients. Am J Kidney Dis 2007;50:594-601.

12. Linden M, Baumann K, Lieberei B, Rotter M. The post-traumatic embitterment disorder self-rating scale (PTED Scale). Clin Psychol Psychother 2009;16:139-147.

13. Martin A, Rief W, Klaiberg A, Braehler E. Validity of the Brief Patient Health Questionnaire Mood Scale (PHQ-9) in the general population. Gen Hosp Psychiatry 2006;28:71-77.

14. Löwe B, Unützer J, Callahan CM, Perkins AJ, Kroenke K. Monitoring depression treatment outcomes with the patient health questionnaire-9. Med Care 2004;42:1194-1201.

15. Kroenke K, Spitzer RL, Williams JB. The PHQ-9: validity of a brief depression severity measure. J Gen Intern Med 2001:16:606-613.

16. Pinto-Meza A, Serrano-Blanco A, Peñarrubia MT, Blanco E, Haro JM. Assessing depression in primary care with the PHQ-9: can it be carried out over the telephone? J Gen Intern Med 2005:20:738-742.

17. Han C, Jo SA, Kwak JH, Pae CU, Steffens D, Jo I, et al. Validation of the Patient Health Questionnaire-9 Korean version in the elderly population: the Ansan Geriatric study. Compr Psychiatry 2008;49:218-223.

18. Yoon S, Lee Y, Han C, Pae CU, Yoon HK, Patkar AA, et al. Usefulness of the Patient Health Questionnaire-9 for Korean medical students. Acad Psychiatry 2014;38:661-667.

19. Han H, Wang SM, Han C, Lee SJ, Pae CU. The relationship between somatic symptoms and depression. Neuro Endocrinol Lett 2014;35:463-469.

20. Sakthong P, Kasemsup V. Health utility measured with EQ-5D in Thai patients undergoing peritoneal dialysis. Value Health 2012;15(1 Suppl):S79-S84.

21. Räsänen $P$, Roine $E$, Sintonen $H$, Semberg-Konttinen $V$, Ryynänen OP, Roine R. Use of quality-adjusted life years for the estimation of effectiveness of health care: a systematic literature review. Int J Technol Assess Health Care 2006:22:235-241.

22. Linden $M$, Baumann K, Lieberei B, Lorenz C, Rotter M. Treatment of posttraumatic embitterment disorder with cognitive behaviour therapy based on wisdom psychology and hedonia strategies. Psychother Psychosom 2011;80:199-205. 